\title{
Effects of Coconut Oil on Human Health
}

\author{
Laura Boemeke1, Aline Marcadenti2,3, Fernanda Michielin Busnello², \\ Catarina Bertaso Andreatta Gottschall2* \\ ${ }^{1}$ Postgraduate Studies Program in Hepatology, Universidade Federal de Ciências da Saúde de Porto Alegre \\ (UFSCPA), Porto Alegre, Brazil \\ ${ }^{2}$ Department of Nutrition, Universidade Federal de Ciências da Saúde de Porto Alegre (UFSCPA), Porto Alegre, \\ Brazil \\ ${ }^{3}$ Postgraduate Studies Program in Cardiology, Instituto de Cardiologia/Fundação Universitária de Cardiologia \\ do Rio Grande do Sul (IC/FUC), Porto Alegre, Brazil \\ Email: ${ }^{*}$ catarina@ufcspa.edu.br
}

Received 30 June 2015; accepted 25 July 2015; published 29 July 2015

Copyright (C) 2015 by authors and Scientific Research Publishing Inc.

This work is licensed under the Creative Commons Attribution International License (CC BY).

http://creativecommons.org/licenses/by/4.0/

(c) (i) Open Access

\begin{abstract}
Coconut oil ( $\mathrm{CO}$ ) has generated discussions about its possible effects on health, especially for being an oil rich in saturated fat, which is known to contribute to the development of atherosclerosis and cardiovascular diseases. On the other hand, $C O$ contains high levels of lauric acid that is directly absorbed by enterocytes and may prevent the fat deposition in blood vessels. In addition, flavonoids and polyphenols present in $\mathrm{CO}$ may be beneficial in reducing the oxidative stress involved in the etiology of various diseases, for instance, cardiovascular diseases and cancer. This article aimed to review the likely benefits of $\mathrm{CO}$ and its effects related to oxidative stress, hyperlipidemia and chronicle diseases in humans.
\end{abstract}

\section{Keywords}

Coconut Oil [Supplementary Concept], Oxidative Stress, Lipids, Dietary Fats

\section{Introduction}

Coconut oil (CO) is composed predominantly of saturated fatty acids (SFA), corresponding to approximately $90 \%$ of its total composition. In nutritional terms, a tablespoon of coconut oil (13 g) contains about $120 \mathrm{kcal}, 12 \mathrm{~g}$ of total fats, $11.2 \mathrm{~g}$ of SFA, $0.7 \mathrm{~g}$ of monounsaturated fatty acids (MUFA) and $0.2 \mathrm{~g}$ of polyunsaturated fatty acids (PUFA) [1]. The main fatty acids (FA) found in CO are the lauric (12:0), myristic (14:0) and palmitic (16:0) acids, which represent $46 \%, 17 \%$ and $9 \%$ of the FA, respectively. Unlike the long-chain FAs, which require the aid of lipoproteins that can be deposited in various organs [2], most of the FAs in CO are composed by medium

${ }^{*}$ Corresponding author. 
chain; thus, they are directly absorbed by the intestine and sent to the liver to be used as an energy source [3], avoiding the stock in adipose tissue and the deposition in other organs such as the heart and the liver. Furthermore, the amount of cholesterol present in this oil is very small: $0.012 \mathrm{mg}$ of dietary cholesterol for each $85 \mathrm{~g}$ of $\mathrm{CO}[2]$.

Most of coconut oil available is derived from the dried pulp of coconut, called copra, which is obtained by drying at low temperatures or dried in the sun. It is possible to combine these two drying techniques to obtain the coconut oil. Then, the oil extraction occurs by pressing process, which yields a crude oil that is subsequently bleached and deodorized.

The use of CO has generated discussion regarding its possible effects on health due especially to its composition, since SFA may contribute to atherosclerosis and consequently, for the development of cardiovascular diseases. However, although being composed largely of SFA, CO has a significant amount of lauric acid, which may avoid the fat deposition in organs and blood vessels and, therefore, it is not considered as a potential atherogenic fat [1]. In addition, flavonoids and polyphenols present in CO may have a beneficial effect regarding the improvement of oxidative stress, involved in the etiology of various diseases, including type-2 diabetes mellitus (T2DM), cardiovascular disease and cancer.

Despite all possible beneficial effects of CO, most of the studies have been conducted in animal models and the literature is scarce about studies in human beings. Therefore, the aim of this article is to review the potential benefits of $\mathrm{CO}$ evaluating its effects related to oxidative stress and chronicle disease in humans.

\section{Oxidative Stress and Lipid Profile}

Oxidative stress is an imbalance between antioxidants and pro-oxidants and it may contribute for the pathogenesis of cardiovascular disease. Flavonoids and other polyphenols present in CO may be responsible for its antioxidant activity [4]. Sabitha et al. [5] evaluated the lipid profile and oxidative stress in men aged 35 to 65; subjects were divided into 4 groups of 35 individuals each: two groups consisting of healthy individuals who consumed soybean oil (SO) or CO, and other two groups consisting of individuals with T2DM, who consumed also $\mathrm{SO}$ or CO. At the end of the study, diabetic subjects who consumed SO or CO had their levels of serum triglycerides, LDL-cholesterol and VLDL-cholesterol increased when compared to healthy individuals; subjects with T2DM had also their levels of total cholesterol and HDL-cholesterol increased after SO intake when compared to healthy subjects. There was no significant difference in lipid profile between the two groups of healthy individuals or between the two groups of diabetic subjects after SO or CO intake. Regarding oxidative stress, individuals with T2DM who consumed SO had their levels of glutathione and oxidized glutathione decreased when compared to healthy individuals who consumed SO; diabetic subjects had also their levels glutathione, oxidized glutathione and superoxide dismutase reduced after CO intake, when compared to healthy individuals. Overall, there was no significant difference in markers of oxidative stress between SO and CO groups.

It is known that T2DM per se leads to alterations in biochemical parameters and induces oxidative stress; therefore, this study is inconclusive about the possible effects of CO regarding changes in lipid profile or in the endogenous antioxidant system. In addition, diabetic subjects were older and some influence in lipid and oxidative stress profile may be due the age.

\section{Obesity and Lipid Profile}

Most of studies conducted in humans regarding the use of CO have evaluated lipid profile and nutritional status. Some of these studies [6] [7] have compared the consumption of CO with SO. Noteworthy is the fact that soybean oil is a major source of PUFAs linoleic (omega-6) and alpha-linolenic (omega-3) fatty acids, while coconut oil is a major source of saturated fat (lauric acid).

Obesity (mainly visceral) is a traditional and independent risk factor for the developing of cardiovascular disease [8]. The effects of $\mathrm{CO}$ on abdominal obesity were evaluated in 40 women aged between 20 and 40 years, who were randomized to consume $30 \mathrm{ml}$ of $\mathrm{CO}$ or $30 \mathrm{ml}$ of SO a day over a 12-week period, during which all subjects were instructed to follow a balanced hypocaloric diet and to walk for $50 \mathrm{~min} / \mathrm{day}$. At the end of the study, CO showed higher levels of HDL-cholesterol ( $48.7 \pm 2.4$ vs. $45.00 \pm 5.6 ; \mathrm{P}=0.01)$ and a lower LDL: HDL ratio (2.41 \pm 0.8 vs. $3.1 \pm 0.8 ; \mathrm{P}=0.04)$. Reductions in body mass index (BMI) were detected in both groups, but only $\mathrm{CO}$ group showed a significant reduction in waist circumference values. Women allocated to SO group had their levels of total cholesterol, LDL-cholesterol and LDL: HDL ratio increased, whilst HDL- 
cholesterol diminished; such alterations were not observed in CO group [6].

In other study, Liau et al. [9] showed a significant reduction in waist circumference among 20 healthy who consumed $30 \mathrm{ml}$ a day of $\mathrm{CO}$ (30 minutes before the meals) over a 4-week period. However, stratifying the sample by gender, a significant reduction in waist circumference was detected only among men. Regarding biochemical parameters, there were no significant changes in levels of total cholesterol and its fractions, but a decrease in creatinine and alanine aminotransferase values. Noteworthy is the fact that despite these positive results, the short period of follow-up and the lack of blinding may be important limitations and it suggest caution in interpretation these findings.

An observational study conducted with 1839 healthy Philippine women detected a higher intake of CO predominantly consumed in the form of frying among women with increased caloric intake, and it was significantly related to increased levels of HDL-cholesterol (CO consumption was not significantly associated with LDLcholesterol or triglyceride values) [10]. Despite the possible beneficial effect of CO regarding increased levels of HDL-cholesterol, external factors and confounding variables may be influenced these results (levels of physical exercise, for instance, were not tested in this study). Further, CO may have its chemical properties altered after frying, influencing negatively some biochemical parameters when consumed in high quantities.

Among 25 healthy men aged 20 to 26 years who consume SO over an 8-week period followed by CO consumption over more 8-week period (3-week washout) it was detected no significant difference in total cholesterol, LDL-cholesterol and HDL-cholesterol levels at the end of CO consumption when compared to SO intake; however, triglyceride values were higher after $\mathrm{CO}$ consumption [7]. In other non-randomized trial conducted among 32 individuals with hypercholesterolemia and supplemented with $30 \mathrm{ml} /$ day of extra virgin CO during 12-weeks, Miranda et al. [11] showed a significant reduction in body weight, BMI, waist circumference and waist-to-hip ratio after intervention. Furthermore, it was found a significant reduction in VLDL-cholesterol, triglycerides and C-reactive protein levels, and increased levels of apolipoprotein A. Moderately hyperlipidemic men and women were also allocated by Cox et al. [12] to consume three different types of fat (butter, CO and safflower) for six months. Authors showed that total cholesterol and LDL-cholesterol were significantly higher on the diet containing butter, respectively than on the CO diet. Levels of apolipoprotein B and A-I were significantly higher on CO and on butter than on safflower oil. HDL-cholesterol did not differ significantly on the three diets whereas levels in women on the butter and CO diets were significantly higher than on the safflower oil diet. Finally, 32 individuals with established coronary heart disease and 16 age and sex matched healthy controls were studied in India; it was concluded that the consumption of coconut and $\mathrm{CO}$ was found to be similar in both groups, and they did not differ in the fat, SFA and dietary cholesterol consumption. Authors suggest no specific role for coconut or CO in the causation of cardiovascular disease in Indian population [13].

\section{Cancer}

One study has evaluated the supplementation of virgin CO in patients with breast cancer, stages III-VI and submitted to 6 cycles of chemotherapy. Women were randomized to an intervention group (10 $\mathrm{ml}$ of CO twice daily after 1 week each chemotherapy cycle from the third until the sixth cycle) or to the control group, who received no supplement. It was found significant mean score differences for functioning and global quality of life between groups; the intervention group also had better scores for symptoms including fatigue, dyspnea, sleep difficulties, and loss of appetite compared to the control group. Although there are deteriorations for sexual enjoyment, the intervention group exhibited improvement in breast functioning and symptom scores for body image, sexual function, future perspective, breast symptoms, and systemic therapy side effects [14]. Despite these interesting results, the study could be subject to reporting bias because it was not clear whether the researcher who applied the questionnaire was blinded to the intervention/control group.

\section{Conclusion}

Although some studies have identified beneficial effects of the CO consumption in human health, there are few data available and most of studies have limitations. Despite positive relation detected in animal models regarding CO and improvement of lipid profile, nutritional status and antioxidant capacity [15]-[17], studies conducted in humans are inconclusive; the small sample size or the allocation of specific groups for the clinical studies (diabetic subjects, individuals with established hyperlipidemia or cardiovascular disease), for instance, makes the generalization of the results difficult. The short time of follow-up in some studies is other important issue. 
The effects of long-term consumption of CO are unknown, except in studies among populations whose main source of dietary fat is CO. Furthermore, studies have assessed predominantly the consumption of CO compared to SO, and it is necessary of further researches regarding the evaluation of the potential CO benefits when compared to other types of fats. Well-designed randomized clinical trials should be conducted to elucidate the possible benefits of CO in human health.

\section{References}

[1] Dayrit, C.S. (2003) Coconut Oil: Atherogenic or Not? (What Therefore Causes Atherosclerosis?). Philippine Journal of Cardiology, 31, 97-104.

[2] Vasudevan, D.M. (2013) Coconut Oil and Health Controversy. International Journal of Health and Rehabilitation Sciences, 2, 157-164.

[3] DebMandal, M. and Mandal, S. (2011) Coconut (Cocos nucifera L.: Arecaceae): In Health Promotion and Disease Prevention. Asian Pacific Journal of Tropical Medicine, 4, 241-247. http://dx.doi.org/10.1016/S1995-7645(11)60078-3

[4] Norliana, M., Kamisah, Y., Nursyafiza, M., Mohd, H. and Qodriyah, S. (2014) Potential Role of Virgin Coconut Oil in Reducing Cardiovascular Risk Factors. Experimental \& Clinical Cardiology, 20, 3399-3410.

[5] Sabitha, P., Vaidyanathan, K., Vasudevan, D.M. and Kamath, P. (2009) Comparison of Lipid Profile and Antioxidant Enzymes among South Indian Men Consuming Coconut Oil and Sunflower Oil. Indian Journal of Clinical Biochemistry, 24, 76-81. http://dx.doi.org/10.1007/s12291-009-0013-2

[6] Assunção, M.L., Ferreira, H.S., dos Santos, A.F., Cabral, C.R. and Florêncio, T.M.M.T. (2009) Effects of Dietary Coconut Oil on the Biochemical and Anthropometric Profiles of Women Presenting Abdominal Obesity. Lipids, 44, 593601. http://dx.doi.org/10.1007/s11745-009-3306-6

[7] Lanka, S. and Studies, F. (1990) The Effect of Daily Consumption of Coconut Fat and Soya-Bean Fat on Plasma Lipids and Lipoproteins of Young Normolipidaemic Men. British Journal of Nutrition, 63, 547-552. http://dx.doi.org/10.1079/BJN19900141

[8] Gondim, R., Helena, M., Benício, D.A., Dias, R. and Latorre, D.O. (2001) Waist Circumference and Body Mass Index as Predictors of Hypertension. Arquivos Brasileiros de Cardiologia, 87, 462-470.

[9] Liau, K.M., Lee, Y.Y., Chen, C.K. and Rasool, A.H.G. (2011) An Open-Label Pilot Study to Assess the Efficacy and Safety of Virgin Coconut Oil in Reducing Visceral Adiposity. International Scholarly Research Notices Pharmacology, 2011, 1-7. http://dx.doi.org/10.5402/2011/949686

[10] Feranil, A.B., Duazo, P.L., Kuzawa, C.W. and Adair, L.S. (2012) Coconut Oil Predicts a Beneficial Lipid Profile in Pre-Menopausal Women in the Philippines. Asia Pacific Journal of Clinical Nutrition, 20, 190-195.

[11] Silva, R.S.M., Fortes, R.C. and Soares, H.F. (2011) Effects of Dietary Supplementation with Coconut Oil on Lipid Profile and Cardiovascular of Dyslipidemic Individuals. Brasília Médica, 48, 42-49.

[12] Cox, C., Mann, J., Sutherland, W. and Chiisholm, A. (1995) Effects of Coconut Oil, Butter, and Safflower Oil on Lipids and Lipoproteins in Persons with Moderately Elevated Cholesterol Levels. The Journal of Lipid Research, 36, 1787-1795.

[13] Kumar, P.D. (1997) The Role of Coconut and Coconut Oil in Coronary Heart Disease in Kerala, South India. Tropical Doctor, 27, 215-217.

[14] Law, K.S., Azman, N., Omar, E.A., Musa, M.Y., Yusoff, N.M., Sulaiman, S.A. and Hussain, N.H.N. (2014) The Effects of Virgin Coconut Oil (VCO) as Supplementation on Quality of Life (QOL) among Breast Cancer Patients. Lipids in Health and Disease, 13, 139. http://dx.doi.org/10.1186/1476-511X-13-139

[15] Nevin, K.G. and Rajmohan, T. (2006) Virgin Coconut Oil Supplemented Diet Increases the Antioxidant Status in Rats. Food Chemistry, 99, 260-266. http://dx.doi.org/10.1016/j.foodchem.2005.06.056

[16] Hostmark, A.T., Spydevold, O. and Eilertsen, E. (1980) Plasma Lipid Concentration and Liver Output of Lipoproteins in Rats Fed Coconut Fat or Sunflower Oil. Artery, 7, 367-383.

[17] Cleary, M.P., Phillips, F.C. and Morton, R.A. (1999) Genotype and Diet Effects in Lean and Obese Zucker Rats Fed Either Safflower or Coconut Oil Diets. Proceedings of the Society for Experimental Biology and Medicine, 220, 153161. http://dx.doi.org/10.3181/00379727-220-44358 